\title{
Repeated Homotypic Stress Elevates 2-Arachidonoylglycerol Levels and Enhances Short-Term Endocannabinoid Signaling at Inhibitory Synapses in Basolateral Amygdala
}

\author{
Sachin Patel*,', Philip J Kingsley ${ }^{2}$, Ken Mackie ${ }^{3}$, Lawrence J Marnett ${ }^{2}$ and Danny G Winder ${ }^{4}$ \\ 'Department of Psychiatry, Vanderbilt University, Nashville, TN, USA; 'Department of Biochemistry, Chemistry, and Pharmacology, Vanderbilt \\ University, Nashville, TN, USA; ${ }^{3}$ Department of Psychological and Brain Sciences, The Linda and Jack Gill Center, Indiana University, Bloomington, \\ IN, USA; ${ }^{4}$ Department of Molecular Physiology and Biophysics, Vanderbilt University, Nashville, TN, USA
}

\begin{abstract}
Psychosocial stress is a risk factor for development and exacerbation of neuropsychiatric illness. Repeated stress causes biochemical adaptations in endocannabinoid (eCB) signaling that contribute to stress-response habituation, however, the synaptic correlates of these adaptations have not been examined. Here, we show that the synthetic enzyme for the eCB 2-arachidonoylglycerol (2-AG), diacylglycerol (DAG) lipase alpha, is heterogeneously expressed in the amygdala, and that levels of 2-AG and precursor DAGs are increased in the basolateral amygdala (BLA) after 10 days, but not I day, of restraint stress. In contrast, arachidonic acid was decreased after both I and 10 days of restraint stress. To examine the synaptic correlates of these alterations in 2-AG metabolism, we used wholecell electrophysiology to determine the effects of restraint stress on depolarization-induced suppression of inhibition (DSI) in the BLA. A single restraint stress exposure did not alter DSI compared with control mice. However, after I0 days of restraint stress, DSI duration, but not magnitude, was significantly prolonged. Inhibition of 2-AG degradation with MAFP also prolonged DSI duration; the effects of repeated restraint stress and MAFP were mutually occlusive. These data indicate that exposure to repeated, but not acute, stress produces neuroadaptations that confer BLA neurons with an enhanced capacity to elevate 2-AG content and engage in 2-AG-mediated short-term retrograde synaptic signaling. We suggest stress-induced enhancement of eCB-mediated suppression of inhibitory transmission in the BLA could contribute to affective dysregulation associated with chronic stress.

Neuropsychopharmacology (2009) 34, 2699-2709; doi:10.1038/npp.2009.101; published online I2 August 2009
\end{abstract}

Keywords: endocannabinoid; CBI receptor; anxiety; depression; marijuana; diacylglycerol lipase

\section{INTRODUCTION}

Psychosocial stress has been shown to contribute to depressive and anxious symptoms in patients with affective illness (Caspi et al, 2003; Hammen, 2005; Hammen et al, 2004; Melchior et al, 2007). Environmental and psychological stressors can recapitulate biochemical, structural, and behavioral aspects of depressive illness in laboratory animals (see Pittenger and Duman, 2008 for review). Recent studies have pointed to the endocannabinoid (eCB) system as an important modulator of stress responses and emotional regulation (Hill and Gorzalka, 2005; Moreira and Lutz, 2008; Patel and Hillard, 2008; Viveros et al, 2005). In addition, the type-1 cannabinoid receptor $\left(\mathrm{CB}_{1}\right)$ gene variants modulate the vulnerability to develop depressive

*Correspondence: Dr S Patel, Department of Psychiatry, Vanderbilt University, Vanderbilt Psychiatric Hospital, 1601 23rd Ave South, Nashville, TN 37212, USA, Tel: + I 615327 7080, Fax: + I 615322 1901, E-mail: sachin.patel@vanderbilt.edu

Received I April 2009; revised 9 June 2009; accepted 9 July 2009 symptoms following stressful life events (Juhasz et al, 2009). Taken together, these data suggest an interaction between stress, eCB signaling, and mental illness. Understanding the neuroadaptations that occur in the eCB system in response to chronic stress could shed light on the neural mechanisms subserving stress-induced exacerbation of affective disorders in humans.

The neuronal eCB signaling system is composed of eCB ligands, including 2-AG and anandamide, their synthetic and catabolic enzymes, and $\mathrm{CB}_{1}$ receptors (Kano et al, 2009; Piomelli, 2003). 2-arachidonoylglycerol (2-AG) is the most abundant $\mathrm{eCB}$ found in brain, and is synthesized in response to Gq-coupled receptor activation or calcium influx, from sn-2 arachidonic acid (AA) containing diacylglycerol (DAG) precursors (Jung et al, 2005, 2007). This synthetic reaction is catalyzed by DAG lipase (DAGL), which is expressed within postsynaptic neuronal elements (Katona et al, 2006; Uchigashima et al, 2007). On synthesis, 2-AG diffuses to presynaptic axon terminals where it activates $\mathrm{CB}_{1}$ receptors to reduce GABA and glutamate release (Kano et al, 2009). 2-AG is then degraded by 
presynaptically located monoglyceride lipase (MGL), to free AA and glycerol (Ahn et al, 2008). This form of retrograde eCB signaling has been studied in several brain regions implicated in the regulation of memory and emotion including the amygdala (Azad et al, 2004; Marsicano et al, 2002; Zhu and Lovinger, 2005).

The amygdaloid complex is a key region subserving stress responses and emotional behavior (Sah et al, 2003) and dysregulation of GABAergic signaling in the basolateral complex of the amygdala has been suggested to contribute to the pathophysiology of affective disorders (Quirk and Gehlert, 2003). Thus, this region is a candidate structure subserving the interactions between stress and exacerbation of mental illness. The basolateral complex contains high levels of $\mathrm{CB}_{1}$ receptors (Katona et al, 2001), and has been shown to express functional eCB signaling at inhibitory synapses (Marsicano et al, 2002; Zhu and Lovinger, 2005). We have earlier shown that restraint stress causes an increase in the concentration of 2-AG within the amygdaloid region (Patel et al, 2005b; Rademacher et al, 2008); an effect that exhibits sensitization on repeated exposure. Stress-induced activation of eCB signaling also has an important role in habituation of active and innate stressresponse behaviors (Kamprath et al, 2006; Patel et al, 2005b). These data suggest that adaptations in 2-AG signaling in the amygdala could contribute to behavioral and emotional alterations that occur in response to chronic stress (Patel and Hillard, 2008), however, the synaptic mechanisms subserving these effects are not known.

Here, we examine the effects of repeated restraint stress on the 2-AG metabolism specifically in the basolateral amygdala (BLA), and the synaptic correlates of stressinduced elevations in 2-AG content in this region. Our data indicate that 2-AG and its DAG precursors are increased within the basolateral subregion of the amygdala after repeated restraint stress. Furthermore, repeated restraint stress prolongs 2-AG-mediated retrograde signaling at inhibitory synapses in this region.

\section{METHODS}

\section{Materials}

Male ICR mice 5-7 weeks of age were used for all experiments (Harlan, Indianapolis, IN). Mice were housed on a 12:12 light-dark cycle (lights on at 06:00), with food and water available ad libitum. All studies were carried out in accordance with the National Institute of Health Guide for the Care and Use of Laboratory Animals.

$N$-(piperidin-1-yl)-5-(4-iodophe-nyl)-1-(2,4-dichlorophenyl)4-methyl-1 H-pyrazole-3-carboxamide (AM251), and $R-(+)-(2$, 3-dihydro-5-methyl-3-[(4morpholinyl)methyl]pyrol[1,2,3-de]1,4-benzoxazin-6-yl)(1-naphthalenyl) methanone monomethanesulfonate (WIN55212-2), and (5Z,8Z,11Z,14Z)-5,8,11, 14-eicosatetraenyl-methyl ester phosphonofluoridic acid (MAFP) were obtained from Tocris (Ellisville, MO). Tetrahydrolipstatin (THL) and essentially fatty-acid free bovine serum albumin (BSA) were obtained from Sigma (St Louis, $\mathrm{MO})$. 6-cyano-7-nitroquinoxaline-2,3-dione (CNQX) and DL2-amino-5-phosphonovalerate (AP-5) were purchased from Ascent Scientific (Princeton, NJ). All cannabinoids were made up as stock solutions in DMSO; $0.5 \mathrm{~g} / \mathrm{l}$ BSA was added to artificial cerebral spinal fluid (ACSF) to increase solubility and minimize nonspecific binding of lipophilic compounds. Equal amounts of DMSO and BSA were used in control experiments.

1-stearoyl-2-arachidonoylglycerol (SAG), 2-AG, AA, and their deuterated analogs were purchased from Cayman Chemicals (Ann Arbor, MI). N-(3-dimethylaminopropyl)$N^{\prime}$-ethylcarbodiimide (EDC) and 4-(dimethylamino)pyridine (DMAP) were purchased from Aldrich Chemical Company (Milwaukee, WI) and used without further purification. Solvents were HPLC grade and purchased from Fisher Scientific (Pittsburg, PA). 1-oleoyl-2-arachidonoylglycerol (OAG) was synthesized by a reaction of 2-AG and oleic acid using EDC and DMAP (catalyst) in heated toluene $\left(60^{\circ} \mathrm{C}\right)$. The reaction was quenched with water. The organic compounds were extracted with ethyl acetate and the product was purified with preparative HPLC.

\section{Stress Protocol}

Mice were housed four per cage during all experiments. Mice were brought into the restraint room daily and subjected to $1 \mathrm{~h}$ of tube restraint in modified $50 \mathrm{ml}$ conical tubes for 1 day or 10 consecutive days (between 9 and $11 \mathrm{am})$. During this time mice were placed in ventilated animal housing cabinet. On termination of the stressor, mice were placed back in their home cage and returned to the animal care facility housing room. Mice were weighed daily before restraint stress. Control mice were left undisturbed in their home cages except for tail marking at the beginning of the experiment and as needed to maintain identifying marks throughout the 10-day protocol. After each stress episode plastic tubes were washed with soap and water, and then rinsed in $70 \%$ ethanol.

\section{Tissue Preparation and Lipid Extraction}

Control mice or stressed mice were killed by decapitation, the brain rapidly removed, and a $2 \mathrm{~mm}$ block containing the amygdala was made using a coronal brain matrix. Blocks were placed on a glass dish on top of dry ice-cooled metal block. Less than $90 \mathrm{~s}$ elapsed between decapitation and tissue freezing. Once frozen, $1 \mathrm{~mm}$ brain punches were made of the amygdala. Tissue was stored at $-80^{\circ} \mathrm{C}$ until used for tissue extraction. Lipid extraction from amygdala micropunches was carried out exactly as described earlier (Patel et al, 2005b), except that the homogenization volume was spiked with 50 pmol $\mathrm{d}^{8}-2-\mathrm{AG}$, and $500 \mathrm{pmol} \mathrm{d}^{8}-\mathrm{SAG}$, and $1000 \mathrm{pmol} \mathrm{d}^{8}$-AA per sample. Immediately before LC-MS analysis, the samples were reconstituted in $200 \mu \mathrm{l}$ of 9:1 methanol:water (v:v), vortexed, and transferred to autosampler vials.

\section{Mass Spectrometry}

Chromatographic separation of the analytes was achieved on a Phenomenex Synergi Polar-RP column $(7.5 \mathrm{~cm} \times$ $0.2 \mathrm{~cm}, 4 \mu \mathrm{m}$ held at $40^{\circ} \mathrm{C}$ ) using the following gradient: $85 \% \mathrm{~B}$ for $0.5 \mathrm{~min}, \% \mathrm{~B}$ increased to $99 \%$ in $3 \mathrm{~min}$ and held at $99 \%$ for an additional $2 \mathrm{~min}$. The column was re-equilibrated at initial conditions for $3 \mathrm{~min}$ before each injection. The flow rate was $0.5 \mathrm{ml} / \mathrm{min}$. Component A was water and 
B was methanol and each component contained $80 \mu \mathrm{M}$ silver acetate and $0.5 \%$ acetic acid (v:v).

The analytes were detected through single reaction monitoring (as $[\mathrm{M}+\mathrm{Ag}]^{+}$complexes except AA, which is ionized as $\left.[(\mathrm{M}-\mathrm{H})+2 \mathrm{Ag}]^{+}\right)$in the positive ion mode using the following reactions (the mass in parentheses represents the mass of the deuterated internal standard): AA $(\mathrm{m} / z$ $519(527) \rightarrow 409(417)) ; \quad 2-A G \quad(m / z \quad 485(493) \rightarrow 411(419))$; SAG $(\mathrm{m} / z$ 751(759) $\rightarrow 411(419))$ and OAG $(\mathrm{m} / z$ 759 $\rightarrow 411)$. Quantification was achieved through stable isotope dilution for AA, 2-AG, and SAG whereas OAG was quantified relative to SAG-d8. Levels of analytes are given in pmols of analyte per mg wet tissue weight.

\section{Immunohistochemistry}

Immnunohistochemistry and immunofluorescence were performed as described earlier (Patel and Hillard, 2003). Briefly, mice were anesthetized with isoflourane and transcardially perfused with $20 \mathrm{ml}$ of phosphate-buffered saline (PBS) followed by $10 \mathrm{ml}$ of ice-cold $4 \%$ paraformadahyde. Brains were removed and post fixed for $24 \mathrm{~h}$ followed by a $48 \mathrm{~h}$ incubation in $30 \%$ sucrose solution. Frozen sections $30 \mu \mathrm{m}$ thick were cut on a cryostat and stored in an ethylene glycol solution at $-20^{\circ} \mathrm{C}$ until use.

Sections were thoroughly washed in tris-buffered saline (TBS), then incubated in $10 \%$ methanol, $2 \%$ hydrogen peroxide solution in TBS for $30 \mathrm{~min}$ followed by washing and incubation in $4 \%$ normal donkey serum and $0.2 \%$ Triton $\mathrm{X}-100$ in TBS. Sections were incubated in an earlier characterized rabbit-anti DAGL $\propto$ at 1:2000 dilution overnight at room temperature (Katona et al, 2006). Sections were washed and then incubated in biotin-conjugated donkeyanti rabbit at 1:500 for $2 \mathrm{~h}$, followed by HPR-conjugated streptavidin at 1:16000 for $90 \mathrm{~min}$ (Jackson ImmunoResearch, West Grove, PA). Sections were developed using diaminobenzadine substrate with nickel/cobalt heavy metal intensification as described earlier. For immunofluorescence, rabbit-anti DAGL $\propto$ was used at $1: 500$, followed by $2 \mathrm{~h}$ incubation in Cy-3-conjugated donkey-anti rabbit at $1: 250$ for $2 \mathrm{~h}$ (Jackson ImmunoResearch).

For biocytin visualization of recorded neurons, $1 \%$ biocytin (Jackson ImmunoResearch) was included in the internal patch solution. After termination of the experiment (30-60 min), slices were placed in 4\% paraformaldahyde for $24 \mathrm{~h}$. Sections were then washed in PBS for $30 \mathrm{~min}$ and incubated in Cy-3-conjugated streptavidin (Jackson ImmunoResearch) at $1: 500$ dilution for $2 \mathrm{~h}$ at room temperature. Sections were washed and mounted on slides using Aquamount and visualized using confocal microscopy.

Brightfield photomicrographs were obtained using an upright Olympus SZX12 microscope coupled to an Olympus CMAD3 camera using Q-capture software. Confocal images of immunofluorescently stained sections were obtained using a Zeiss LSM510 confocal microscope. Small brightness and contrast adjustments, and figure arrangement were done using Adobe Photoshop CS.

\section{Electrophysiology}

Animals were retrieved from the colony and allowed to rest in sound attenuating boxes for a minimum of $1 \mathrm{~h}$ after which they were killed by decapitation. Stressed animals were killed by cervical dislocation and decapitated immediately following termination of the last $1 \mathrm{~h}$ restraint exposure. A $3 \mathrm{~mm}$ coronal block containing the amygdala was cut using a coronal brain matrix kept on ice; $300 \mu \mathrm{m}$ coronal slices were made on a Leica VT1000S vibratome (Leica Microsystems, Bannockburn, IL) in a $1-4^{\circ} \mathrm{C}$, oxygenated $\left(95 \% \mathrm{O}_{2}, 5 \% \mathrm{CO}_{2}\right), 30 \%$ sucrose, low $\mathrm{Na}^{+}$artificial cerebral spinal fluid containing in $\mathrm{mM}$ : 194 sucrose, 20 $\mathrm{NaCl}, 4.4 \mathrm{KCl}, 2 \mathrm{CaCl}_{2}, 1 \mathrm{MgCl}_{2}, 1.2 \mathrm{NaH}_{2} \mathrm{PO}_{4}, 10$ glucose, 26 $\mathrm{NaHCO}_{3}$. Once cut, sections were transferred to a holding chamber containing oxygenated ACSF in mM: $124 \mathrm{NaCl}$, $4.4 \mathrm{KCl}, 2 \mathrm{CaCl}_{2}, 1.2 \mathrm{MgSO}_{4}, 1 \mathrm{NaH}_{2} \mathrm{PO}_{4}, 10$ glucose, 26 $\mathrm{NaHCO}_{3}$ at $28^{\circ} \mathrm{C}$. After a minimum of $1 \mathrm{~h}$, sections were placed in the recording chamber superfused with oxygenated ACSF at a flow rate of $2 \mathrm{ml} / \mathrm{min}$. To pharmacologically isolate inhibitory postsynaptic currents (IPSCs), ACSF was supplemented with a combination of $10 \mu \mathrm{M}$ CNQX and $50 \mu \mathrm{M}$ D/L AP-5. All experiments were carried out at $23-25^{\circ} \mathrm{C}$.

Patch electrodes (3-6 $\Omega \mathrm{M})$ were pulled on a Flaming/ Brown microelectrode puller (Sutter Instruments) and filled with internal solution containing in $\mathrm{mM}$ : K-gluconate 70 , KCl 80, EGTA 1, HEPES 10, Na-ATP 4, Na-GTP 0.3, QX-314 2 , with osmolarity adjusted to 285-290 mOsm. Visually identified pyramidal neurons within the BLA were used for electrophysiological studies. Recordings were made using an Axopatch 1D amplifier (Axon instruments). Recordings were made at a holding potential of $-70 \mathrm{mV}$. Evoked IPSCs (eIPSCs) were elicited by a bipolar stimulating electrode placed in the BLA with stimulation intensities varied from 3 to $40 \mathrm{~V}$. eIPSP amplitudes were typically adjusted to 150-500 pA. Data were recorded using PClamp 9.2 (Molecular Devices).

For $\mathrm{CB}_{1}$ agonist and antagonist experiments, evoked simulations were elicited at $0.1 \mathrm{~Hz}$ with six stimulations being averaged to obtain one data point per minute. For depolarization-induced suppression of inhibition (DSI) studies, stimulations were made at $0.33 \mathrm{~Hz}$. Access resistance $(\mathrm{Ra})$ was monitored online using a $5 \mathrm{mV}$ voltage step preceding each stimulation. Cells that showed increases in Ra of $>20 \%$ during an experiment or DSI trial or had an $\mathrm{Ra}>35 \mathrm{~m} \Omega$ were excluded from the analysis.

\section{Statistical Analysis}

Statistical analyses were performed using Prism Graphpad (San Diego, CA). Differences in 2-AG, AA, OAG, and SAG were evaluated by ANOVA followed by Dunnett's test post hoc. For drug and stress DSI time-course experiments, data were analyzed by repeated measures ANOVA factoring time and treatment (drug or stress). For DSI summary data, we divided the postdepolarization period into a maximal DSI (average amplitude of the first three eIPSCs after the depolarizing pulse) and a late DSI (average amplitude of the last 10 eIPSCs of the experiment) component, which were compared with the average amplitude of the 10 baseline eIPSCs. Selected comparisons of summary DSI data were analyzed by ANOVA followed by Bonferroni's test post hoc. Typically, data from two to three DSI trials per cell were averaged before analysis. $p<0.05$ was considered significant throughout. Data are presented as mean \pm SEM. 


\section{RESULTS}

\section{Anatomical Distribution of DAGL $\propto$ in the Amygdala}

DAGL synthesizes 2-AG from DAGs, however, the distribution of this enzyme in the amygdala has not been reported. To determine the regional distribution and staining pattern of DAGL $\propto$ we used an earlier characterized antibody directed against an intracellular 118 amino-acid residue sequence of the DAGL $\propto$ protein (Katona et al, 2006). DAGL $\propto$ immunoreactivity was heterogeneously distributed throughout the amygdaloid complex. Dense neuropil staining was evident in the BLA with clear difference in staining intensity evident between subregions of the basolateral complex (Figure 1a). The dorsal division of the lateral nucleus (Ld) and BLA were intensely stained, whereas the ventral division of the lateral amygdala (Lv) showed lower immunoreactivity. Within the central amygdala, the medial division (CeAM) showed less immunoreactivity than the CeAL, but was still above the signal of the adjacent white matter. Increased immunoreactivity was also observed within intercalated cell masses (ICM; Figure 1a). Laser scanning confocal images of the BLA showed neuropil staining surrounding unstained cell bodies (Figure 1b). Higher magnification photomicrographs showed a punctate distribution superimposed on a less intense reticular pattern (Figure 1c).

\section{Effects of Restraint Stress on the 2-AG Metabolism in the BLA}

We have shown earlier that repeated stress increases 2-AG in the amygdaloid region, an effect that shows sensitization such that maximal increases are seen after 10 consecutive days of restraint (Patel and Hillard, 2008; Rademacher et al, 2008). We sought to extend these findings by increasing our anatomical resolution to examine the BLA subregion of the amygdala, and determine the effects of repeated restraint stress on 2-AG precursors and metabolites in this region. We developed an analytical approach that allowed for the simultaneous detection of 2-AG, AA, OAG, and SAG from $1 \mathrm{~mm}$ micropunches of the BLA (mean tissue weight $5.0 \pm 0.2 \mathrm{mg}, N=18$; punch location shown in Figure $2 \mathrm{~A}$ and $\mathrm{B})$. A representative chromatogram depicting retention times and high signal-to-noise ratio for the endogenous compounds, and corresponding deuterated internal standards, are shown in Figure 2C. We stressed mice for either $20 \mathrm{~min}$ or $1 \mathrm{~h}$, or for $1 \mathrm{~h}$ per day for 10 consecutive days. Chronically stressed mice gained significantly less weight over the 10-day restraint protocol than control mice (control $4.4 \pm 0.4 \mathrm{~g} \quad N=8 \quad v s$ stress $-0.1 \pm 0.3 \quad N=12$; $p<0.0001$ by $t$-test). On the last day of restraint exposure (day 1 for acute stress and day 10 for chronic stress), separate groups of mice were killed either 20 or 60 min into the restraint session. In each experiment (acute and chronic restraint exposure) two separate cohorts of mice were used for these two time points with corresponding control groups. Each stress group was normalized to its respective control groups, which were then combined for statistical analysis.

In one representative experiment from control mice, levels of analytes in the BLA were as follows: 2-AG
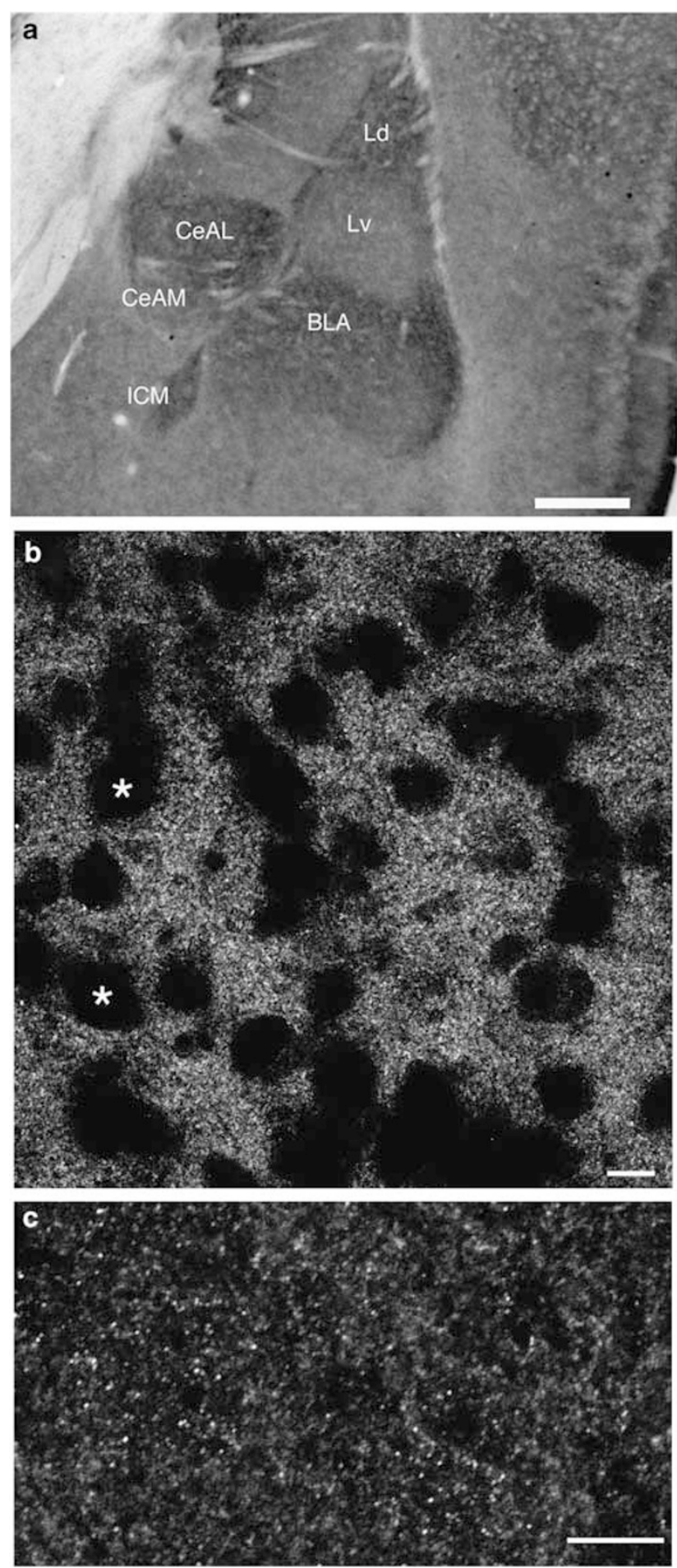

Figure I Anatomical distribution of DAGL $\propto$ in the amygdala. Brightfield photomicrographs of DAGL $\propto$ immunoreactivity in the amygdala (a). (b) High magnification confocal image of DAGL $\propto$ immunofluorescence in the BLA. Note intense neuropil staining surrounding unstained cell bodies (*). (c) High magnification confocal image showing punctate distribution of DAGL $\propto$ in the BLA. Scale bar is $250 \mu \mathrm{m}$ in (a), $25 \mu \mathrm{m}$ in (c), and $15 \mu \mathrm{m}$ in (d).

$26.1 \pm 1.4 \mathrm{pmol} / \mathrm{mg}$, AA $319.1 \pm 25.8 \mathrm{pmol} / \mathrm{mg}, \mathrm{OAG} 11.0 \pm$ $0.5 \mathrm{pmol} / \mathrm{mg}, \mathrm{SAG} 143.7 \pm 8.8 \mathrm{pmol} / \mathrm{mg} ; N=9$. Acute exposure to either 20 or $60 \mathrm{~min}$ of restraint stress did not 

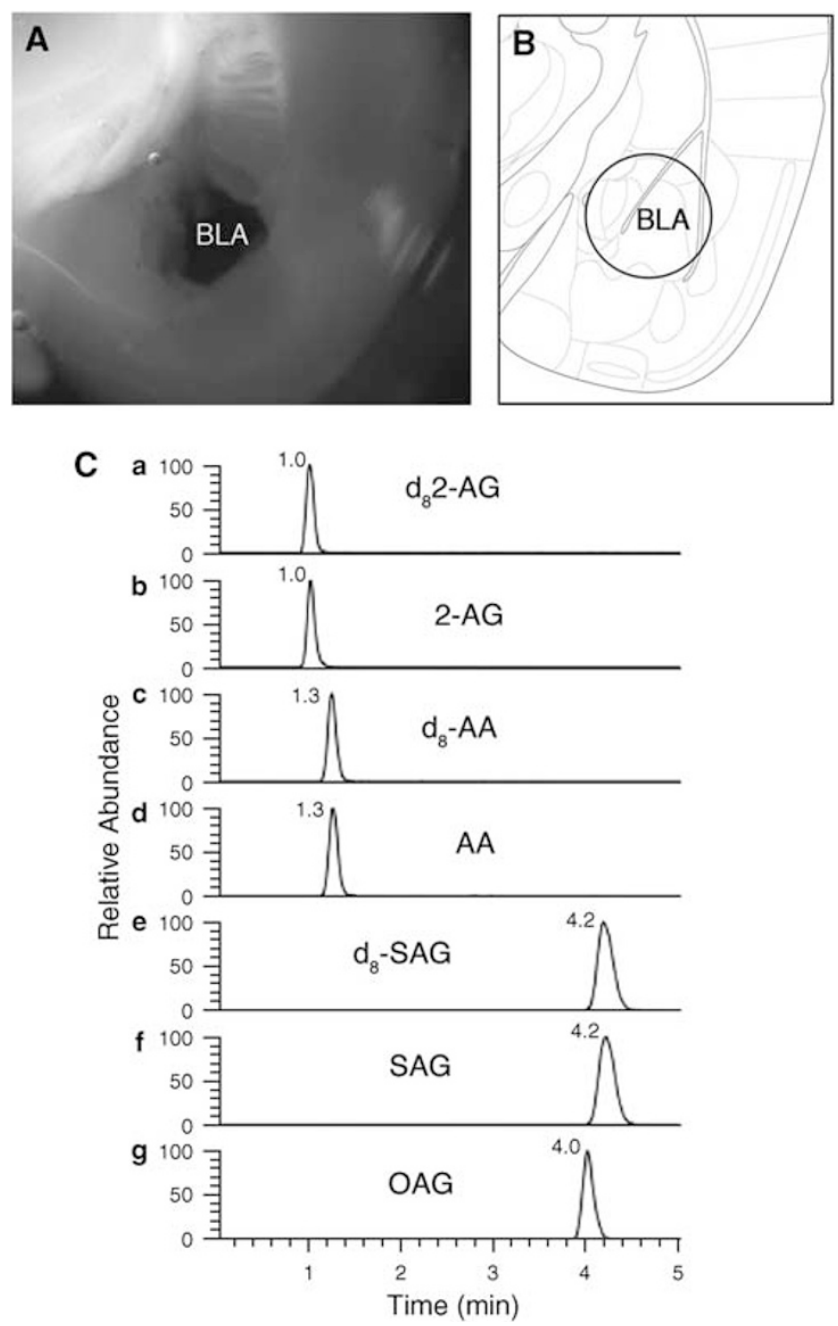

Figure 2 (A) Photomicrograph showing location of micropunches used for eCB analysis. (B) Schematic diagram of I mm micropunch location. (C) Representative chromatogram showing peak detection and retention times of (a) $d_{8}$-AG; (b) endogenous 2-AG; (c) $d_{8}-A A$; (d) endogenous AA; (e) $\mathrm{d}_{8}$-SAG; $(f)$ endogenous SAG; and $(\mathrm{g})$ endogenous OAG. Note high signal to noise ratio of all compounds. Retention times noted above peak.

significantly affect 2-AG levels in the BLA (Figure 3a). AA was decreased after both 20 and 60 min restraint exposures (Figure 3b; $p<0.01$ for both time points). OAG and SAG were unchanged after either 20 or $60 \mathrm{~min}$ of acute restraint stress exposure (Figure $3 \mathrm{c}$ and $\mathrm{d}$ ).

Chronic exposure to restraint stress for 10 consecutive days significantly increased 2 -AG levels $20 \mathrm{~min}(p<0.01)$, but not $60 \mathrm{~min}$ into the 10th restraint session (Figure 3a). AA was decreased at both 20 and $60 \min (p<0.01$ for both time points) into the 10th restraint session (Figure $3 \mathrm{~b}$ ). OAG was significantly increased at both 20 and $60 \mathrm{~min}$ into the 10th restraint episode $(p<0.05$ for both time points; Figure 3c). SAG was increased $60 \mathrm{~min}$ into the 10th restraint episode $(p<0.05$; Figure $3 \mathrm{~d})$.

\section{DSI Is Expressed by BLA Neurons}

Our biochemical data indicate that repeated restraint stress exposure results in a transient increase in $2-\mathrm{AG}$
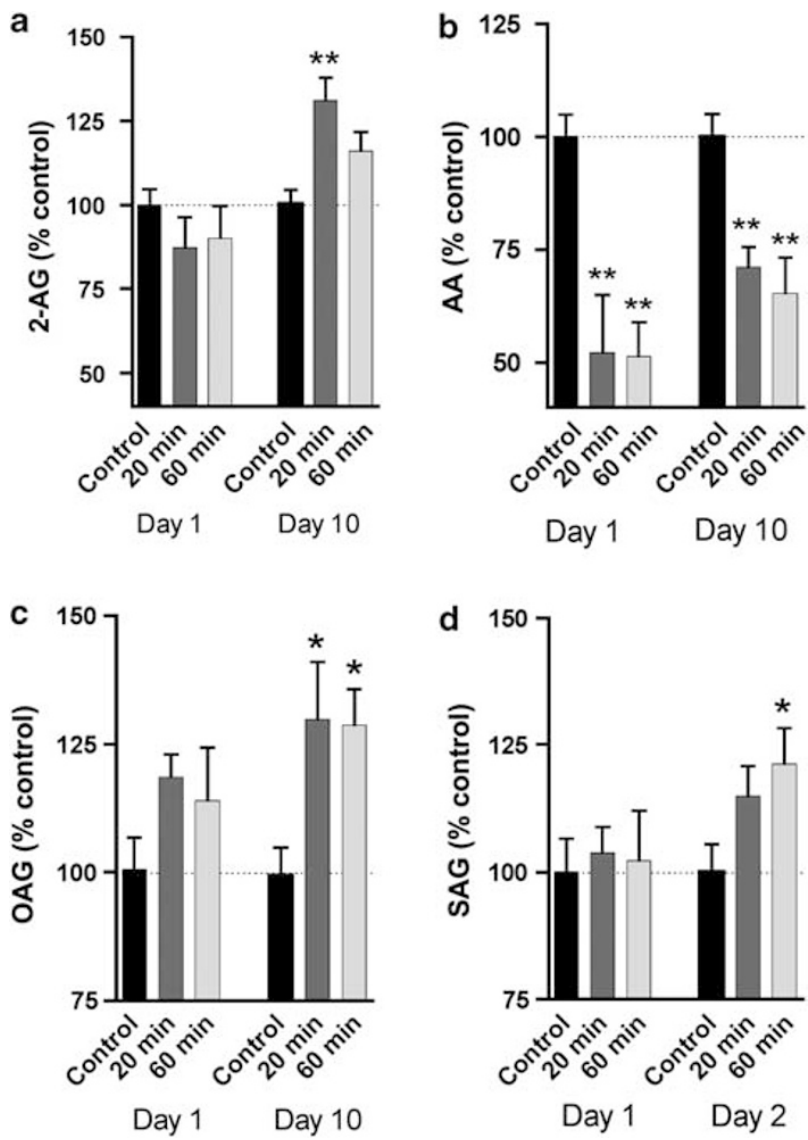

Figure 3 Effects of I and I0 days of restraint stress on (a) 2-AG, (b) AA, (c) OAG, and (d) SAG levels in the BLA. Mice were killed either 20 or $60 \mathrm{~min}$ into the Ist or IOth restraint session. Data are presented as \% control (see text for basal levels). ${ }^{*} p<0.05,{ }^{*} * 00.0$ I significantly different from control.

levels in the BLA. These data, combined with our earlier studies demonstrating a sensitization to stress-induced elevation sin 2-AG (Patel et al, 2005b; Rademacher et al, 2008) suggest adaptations in 2-AG metabolism in the BLA confers BLA neurons with an enhanced ability to transiently maintain elevated levels of 2-AG in response to subsequent restraint exposure. We used whole-cell recordings from BLA neurons to test the hypothesis that this enhanced capacity to increase 2-AG levels in response to restraint stress translates into an enhanced capacity for 2-AGmediated synaptic signaling.

We focused our electrophysiological studies on the BLA given the high level of both DAGL $\propto$ and $\mathrm{CB}_{1}$ receptor expression in this subregion (Katona et al, 2001). Schematic diagram of recording sites and stimulation sites within the BLA are shown in Figure 4a. Recordings were restricted to the magnocellular division of the BLA. A representative biocytin filled neuron typical of cells used for electrophysiological recordings is shown in Figure $4 \mathrm{~b}$. We first examined the ability of BLA principal neurons to engage in eCB-mediated suppression of GABAergic transmission. Earlier data from isolated neurons and brain slices from postnatal rats indicate that $\mathrm{eCB}$ signaling mediates DSI in the BLA (Zhu and Lovinger, 2005), as it does in numerous other brain regions (Kano et al, 2009). Consistent with these 
data we found that postsynaptic depolarization from -70 to $0 \mathrm{mV}$ for $5 \mathrm{~s}$ produced DSI, reducing the IPSC amplitude to $80.1 \pm 5.1 \%$ of control $(p<0.001, N=12$; Figure $4 \mathrm{c})$, which recovered to baseline by $30 \mathrm{~s}$.
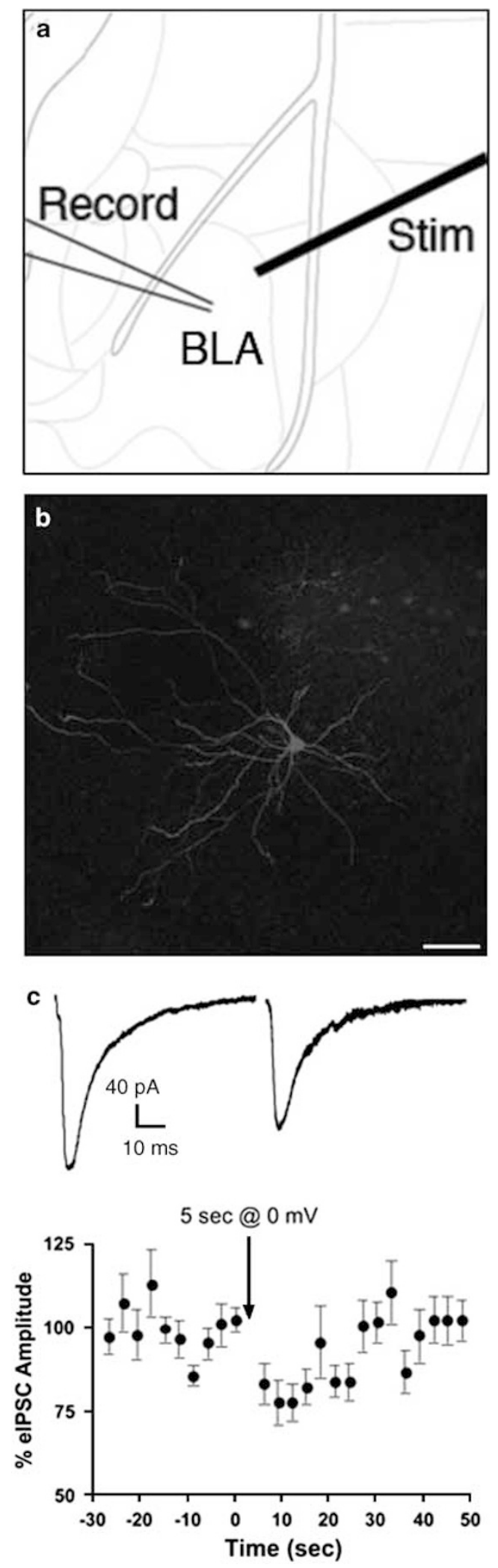

Repeated, but not Acute, Restraint Stress Prolongs DSI in BLA

Given that repeated, but not acute, restraint stress confers an enhanced capacity to elevate 2-AG levels, we determined the effect of acute and repeated restraint stress on DSI in the BLA. Two-way ANOVA showed a significant effect of stress exposure $(p<0.0001)$, and time $(p<0.0001)$, but no significant interaction $(p>0.05)$. Neither acute nor repeated stress affected maximal DSI, but repeated restraint stress prolonged DSI duration (Figure 5a). Summary data indicate that repeated stress did not affect maximal DSI (control $83.5 \pm 4.4 \% \quad N=17 \quad v s$ stress $73.9 \pm 4.8 \quad N=18, p>0.05$; Figure $5 \mathrm{~b}$ ), but significantly enhanced late DSI (control $99.9 \pm 4.0 \% N=17 v s$ repeated stress $80.2 \pm 3.8 \% N=18$, $p<0.01$; Figure $5 b$ ). In contrast, a single $60 \mathrm{~min}$ restraint stress exposure did not significantly affect either maximal
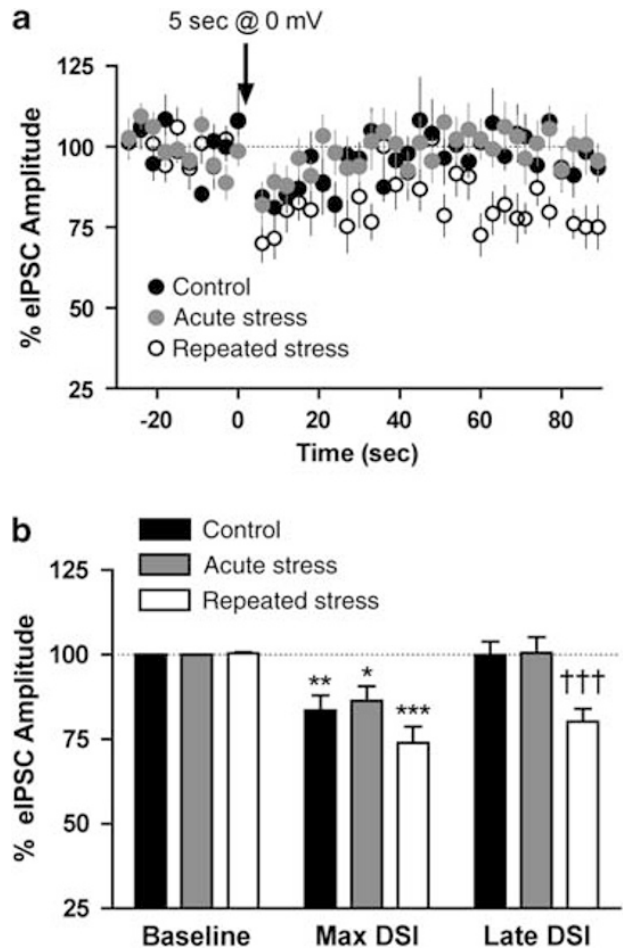

Figure 5 Repeated, but not acute, restraint stress prolongs DSI duration. (a) Effects of 1 or 10 days of restraint stress on the time course of DSI ( $N=18$ per group). (b) Summary data showing neither acute nor repeated restraint stress effects maximal DSI, and only repeated restraint stress significantly enhances late DSI compared with control. * $p<0.05$, ** $p<0.0$ I, **** $p<0.00$ I compared with baseline; ${ }^{\dagger \dagger} p<0.0$ I compared with control late DSI.

Figure 4 (a) Schematic depiction of recording and stimulation sites used for electrophysiological studies. (b) Confocal photomicrograph of biocytin filled pyramidal neuron in the BLA representative of cells used in electrophysiological studies. Note complex dendritic arborization and pyramidal-like soma indicative of BLA pyramidal neurons. Scale bar is $160 \mu \mathrm{m}$. (c) Postsynaptic depolarization to $0 \mathrm{mV}$ for $5 \mathrm{~s}$ causes a transient inhibition of eIPSC amplitude, DSI. Left trace is average of 10 baseline stimulations, right trace is average of three stimulations after depolarization, from a representative neuron. Summary time course is shown below $(N=12)$ 
DSI (control $83.5 \pm 4.4 \% N=17$ vs acute stress $86.3 \pm 4.5 \%$ $N=13, p>0.05$; Figure $5 b$ ) or late DSI (control $99.9 \pm 4.0 \%$ $N=17 v s$ acute stress $100.6 \pm 4.6 N=13, p<0.05$; Figure $5 b$ ) relative to control mice. These data indicate that exposure to repeated, but not acute, restraint stress confers an increased capacity for 2-AG-mediated synaptic signaling in the BLA.

\section{MAFP and Repeated Restraint Stress Produce Mutually} Occlusive Prolongation of DSI

As inhibition of 2-AG hydrolysis with MAFP has been shown to increase DSI duration in the hippocampus (Hashimotodani et al, 2007b), we determined the effects of $1 \mu \mathrm{M}$ MAFP on DSI in the BLA. Bath application of MAFP for $>30 \mathrm{~min}$ before recording significantly prolonged DSI (Figure 6a). Two-way ANOVA showed a significant effect of MAFP $(p<0.001)$, time $(p<0.001)$, and a significant interaction $(p<0.05)$. Summary data indicate that MAFP did not significantly increase maximal DSI compared with vehicle (vehicle $80.1 \pm 5.1 \% \quad N=12$ vs MAFP $66.3 \pm 5.4 \%$ $N=8, p>0.05$ ), but robustly increased the late component of DSI (vehicle $102.5 \pm 4.4 \% N=12$ vs MAFP $74.7 \pm 3.6 \%$ $N=8, p<0.001)$. These data indicate that DSI duration is limited by eCB degradation.

Our data with the MGL inhibitor MAFP indicate that DSI is likely mediated by 2-AG, however, MAFP also inhibits degradation of another eCB, anandamide. To confirm that DSI is mediated by 2-AG in the BLA, we applied the DAGL inhibitor THL in combination with MAFP. In the presence of MAFP, bath application of the DAGL inhibitor THL $10 \mu \mathrm{M}$ for $>30$ min before experiments completely blocked DSI (Figure 6a). Two-way ANOVA showed a significant effect of THL $(p<0.0001)$, time $(p<0.05)$, and a significant interaction $(p<0.01)$. Summary data indicate that THL blocked both maximal DSI (MAFP-vehicle $66.3 \pm 5.4 \% N=8$ $v s$ MAFP + THL $106.4 \pm 7.8 \% N=9, p<0.001$; Figure $6 \mathrm{~b}$ ) and late DSI (MAFP-vehicle $74.7 \pm 3.6 \% N=8$ vs MAFPTHL $104.7 \pm 5.8 \% N=9, p<0.01$; Figure $6 \mathrm{~b}$ ). These data indicate that the ability of MAFP to prolong DSI requires DAGL activity, thus indicating DSI is mediated by $2-A G$ in the BLA.

Both repeated restraint stress and MAFP prolong DSI; if there is a shared mechanism subserving this prolongation we would expect that MAFP and repeated restraint stress would produce a mutually occlusive enhancement of DSI. We therefore tested DSI in mice exposed to 10 days of restraint stress in the presence of MAFP $(1 \mu \mathrm{M})$. Two-way ANOVA showed a significant effect of time $(p<0.0001)$, stress $(p<0.0001)$, but no significant interaction $(p>0.05$; Figure $6 c)$. Summary data indicate maximal DSI was not different between repeated restraint stress $(74.0 \pm 4.8 \%$ $N=18)$, control-MAFP $(66.3 \pm 5.4 \% N=8)$, and repeated restraint stress-MAFP $(67.8 \pm 4.4 \% N=6)$ groups, $p>0.05$ for all comparisons (Figure 6d). Late DSI was also not different between repeated restraint stress $(80.2 \pm 3.8 \%$ $N=18)$, control-MAFP $(74.7 \pm 3.6 \% N=8)$, and repeated restraint stress-MAFP ( $80.0 \pm 7.4 N=6$ groups $), p>0.05$ for
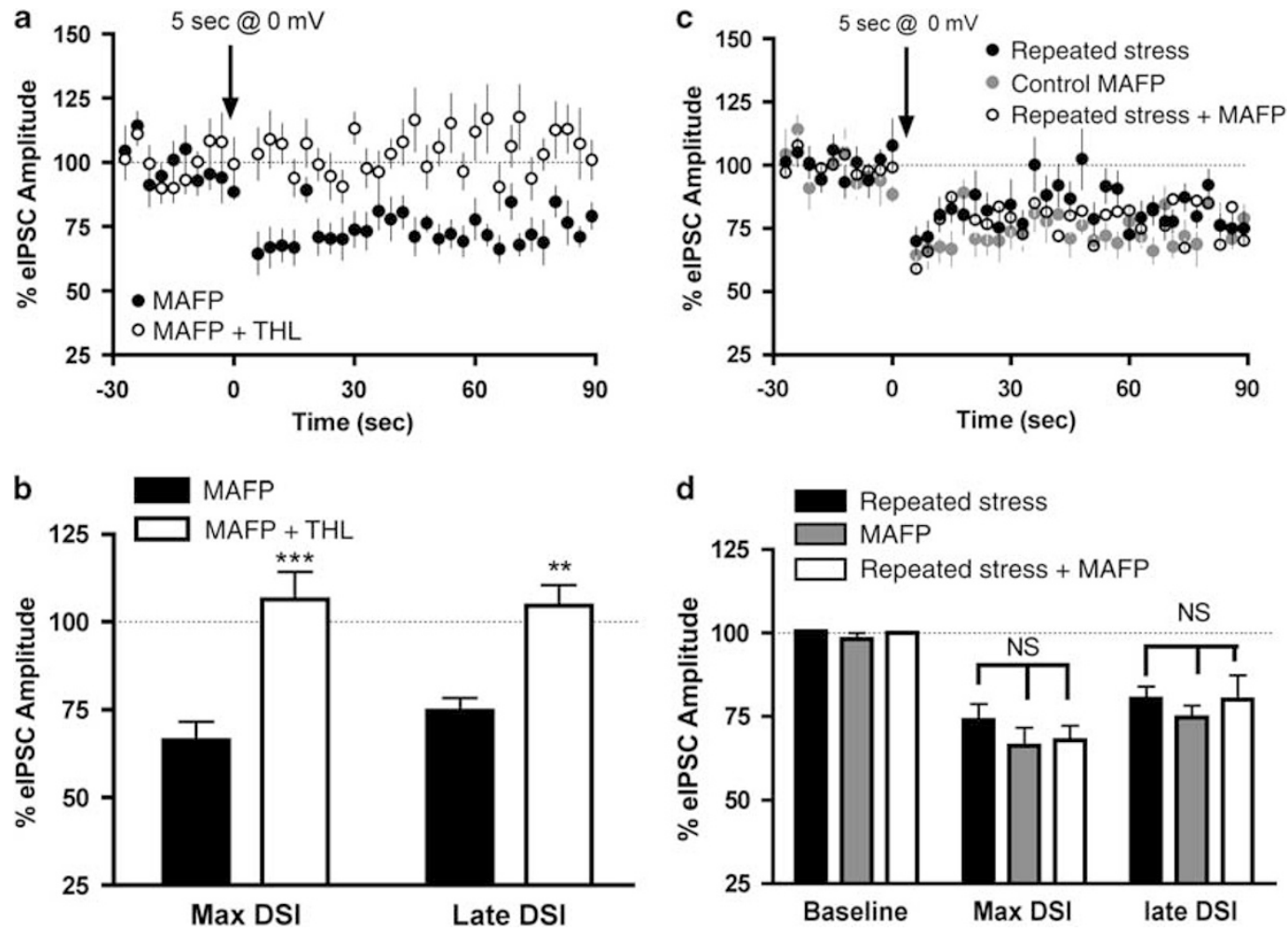

Figure 6 (a) The 2-AG degradation inhibitor MAFP prolongs DSI duration in control cells (solid circles). Co-application of THL and MAFP blocks DSI in BLA neurons (open circles, $N=8-9$ per group). (b) Summary data indicate that MAFP-THL co-application blocks both maximal DSI and late DSI compared with MAFP-vehicle. (c) Time-course data showing repeated restraint stress, control-MAFP, and repeated restraint stress-MAFP groups produce mutually occlusive prolongation of DSI. (d) Summary data showing that neither maximal DSI nor late DSI is different between these three groups. ${ }^{*} * 2<0.0$, ***** $p<0.00$ I compared with MAFP-vehicle. 


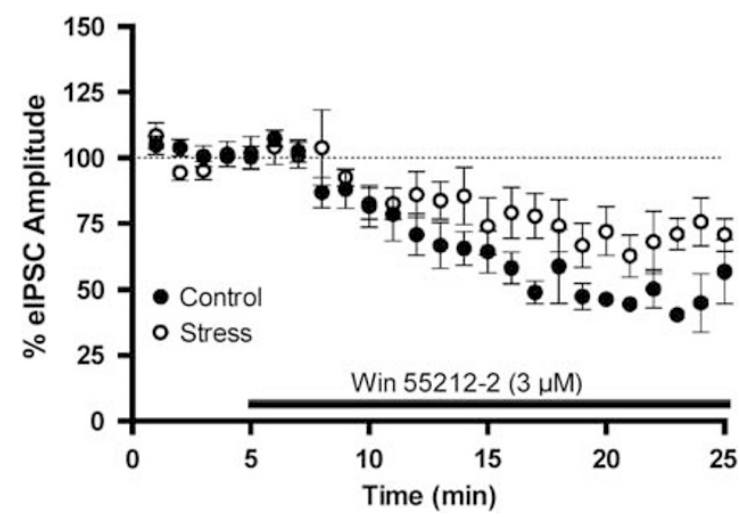

Figure 7 Repeated restraint stress decreases $C_{3}$, receptor sensitivity. Application of Win 552 I2-2 (3 $\mu \mathrm{M}$ ) causes suppression of elPSC amplitude in BLA neurons, but the effect of Win 55212-2 was reduced in mice exposed to 10 days of restraint stress. ANOVA showed significant effect of stress on Win 55212-2-induced suppression of elPSCs, $p<0.000$ I.

all comparisons (Figure 6d). These data suggest a mechanism involving impaired 2-AG degradation could subserve the prolongation of DSI observed after repeated stress exposure.

\section{Stress-Induced DSI Prolongation Is not Mediated by Increase CB1 Receptor Sensitivity}

Although our biochemical data indicate that 2-AG is elevated by repeated restraint stress, it is possible that the enhanced DSI observed in stressed mice could be related to increased $C_{1}$ receptor signaling, rather than increased in eCB production. To examine this possibility directly, we determined the effects of the $\mathrm{CB}_{1}$ receptor agonist Win 55212-2 on GABAergic transmission in control and stressed mice. In control mice, Win 55212-2 suppressed eIPSC amplitude to $47.3 \pm 4.0 \%$ of baseline $(N=5$; Figure 7$)$. In mice exposed to 10 days of restraint stress, Win 55212-2 suppressed eIPSC amplitude to $66.2 \pm 9.1 \%$ of baseline, $N=5$. Two-way ANOVA showed a significant effect of repeated stress $(p<0.0001)$, time $(p<0.0001)$, but no interaction. These data indicate that repeated restraint stress decreases $\mathrm{CB}_{1}$ receptor sensitivity, indicating the enhanced DSI is not mediated increased $C_{1}$ receptor signaling.

\section{DISCUSSION}

The main findings of study are that (1) the 2-AG synthetic enzyme DAGL $\propto$ is heterogeneously expressed in the amygdaloid complex with the highest levels observed in the BLA, CeAL, and ICMs. (2) 2-AG and precursor DAG levels are increased by repeated, but not acute restraint stress exposure, whereas AA levels are reduced by both acute and repeated restraint stress. (3) Exposure to repeated, but not acute, restraint stress enhances DSI duration in BLA neurons. (4) Repeated restraint stress and the 2-AG degradation inhibitor MAFP produce mutually occlusive prolongation of DSI, suggesting a mechanism involving impaired 2-AG catabolism could subserve these effects. These data suggest that repeated stress exposure confers BLA neurons with an enhanced capacity to elevate $2-A G$ in response to subsequent stress exposure, and that this enhanced capacity to elevate 2-AG levels is associated with enhanced 2-AG-mediated short-term synaptic suppression of GABAergic transmission in the BLA.

\section{Stress-Induced Alterations in 2-AG Metabolism}

We have shown earlier that repeated exposure to restraint stress causes a progressive increase in 2-AG content in the amygdaloid region (Patel et al, 2005b; Rademacher et al, 2008). We extend these findings to show that this effect can be observed within the BLA subregion of the amygdala. No increases in 2-AG were observed after acute restraint stress, however, significant increases in 2-AG were observed after 10 consecutive restraint exposures. Furthermore, the effect is transient, with levels returning toward control by the end of the 10th restraint episode. These data suggest that repeated restraint stress confers BLA neurons with an enhanced capacity to transiently elevate 2-AG levels in response to subsequent restraint exposure.

We have also evaluated levels of the 2-AG precursors, OAG, and SAG, after acute and repeated restraint stress. OAG and SAG are likely the predominant DAG species present in neuronal cells, and are depleted by DAGL $\propto$ over expression (Jung et al, 2007), suggesting they serve as precursors for 2-AG synthesis. Our data indicate that both DAGs are elevated by repeated, but not acute, restraint stress. As SAG appeared to increase progressively throughout the $60 \mathrm{~min}$ stress exposure, we suggest that increases in DAGs are triggered by exposure to the 10th restraint episode, rather than an enduring effect initiated by earlier restraint sessions. The increase in $\mathrm{OAG}$ is $\sim 2-3 \mathrm{pmol} / \mathrm{mg}$, whereas the increase in $S A G$ is $\sim 30 \mathrm{pmol} / \mathrm{mg}$. The increase in $2-\mathrm{AG}$ is $\sim 5 \mathrm{pmol} / \mathrm{mg}$, suggesting that increased substrate availability could contribute to the transient increase in 2-AG observed in the same region. The source of increased DAGs is unknown but may be related to increased phospholipase-C activity initiated by upstream Gq-coupled receptor activation.

We also determined levels of the primary metabolite of 2-AG, AA, after acute and repeated restraint stress. Our data indicate that both acute and repeated restraint stress robustly decrease AA levels in the BLA. These data are not surprising because AA is a substrate for cyclooxygenase-2, which is regulated by glucocorticoids (Yamagata et al, 1993). Pharmacological inhibition of MGL has been shown to elevate 2-AG and decrease AA levels, leading some authors to suggest 2-AG degradation by MGL regulates AA levels in vivo (Long et al, 2009; Nomura et al, 2008). However, as 2-AG is only elevated during the 10th restraint exposure, our data suggest that 2-AG degradation under physiological conditions exerts minimal regulation over AA levels in vivo. Furthermore, these data suggest that measurement of free AA does not provide an accurate reflection of 2-AG catabolism under physiological conditions, likely because of the complex regulation of this lipid by multiple metabolic pathways. Although further studies are required to determine the mechanisms subserving the alterations in 2-AG metabolism induced by repeated restraint stress, these data provide the first report of physiological alterations in the 2-AG precursor/metabolite profile in vivo. 


\section{DSI in the BLA}

DSI is a widespread form of eCB-mediated short-term synaptic plasticity expressed by neocortical, allocortical, striatal, midbrain, and cerebellar neurons (Kano et al, 2009). DSI has been recently described in BLA slices and dissociated neurons from postnatal rats (Zhu and Lovinger, 2005). As in other brain regions, DSI in the BLA is blocked by $\mathrm{CB}_{1}$ receptor antagonists and intracellular calcium chelation, indicating mediation by calcium-dependent eCB signaling (Zhu and Lovinger, 2005). We extend these findings to show that DSI is present in the mature mouse BLA; however, the magnitude of suppression $(\sim 20 \%)$ is far less than seen in hippocampus (Wilson and Nicoll, 2001) or cerebellum (Kreitzer and Regehr, 2001), but consistent with data from postnatal rat BLA slices (Zhu and Lovinger, 2005). The relatively small magnitude of DSI in this region is not because of a lack of functional $\mathrm{CB}_{1}$ receptors, because exogenous application of the $\mathrm{CB}_{1}$ agonist Win 55212-2 produced a robust $\sim 50 \%$ suppression of eIPSC amplitude in the BLA, which is consistent with earlier data (Azad et al, 2003; Katona et al, 2001). It is also not because of a general impairment in 2-AG signaling because receptor-driven eCB release initiated by $50 \mu \mathrm{M}$ dihydroxyphenylglycine ( $\sim 50 \%$ eIPSC suppression) is comparable to that observed in BLA and other brain regions (Patel and Winder, unpublished data; (Azad et al, 2004)). Thus, it seems that the small magnitude of DSI is attributable to a specific impairment in calcium-dependent eCB synthesis and/or efflux. It is unlikely that high 2-AG degradation rates contribute to the reduced DSI magnitude because pharmacological blockade of MGL activity did not significantly increase maximal DSI.

Although DSI is mediated by eCB signaling in the BLA (Zhu and Lovinger, 2005), the identity of the eCB ligand remains unknown. We show that DSI is blocked by inhibition of DAGL with THL, indicating mediation by 2-AG. These findings are consistent with earlier data showing that DSI is typically mediated by 2-AG (see Kano et al, 2009 for review). We also show that blockade of MGL with MAFP prolongs DSI in the BLA, consistent with data from hippocampus (Hashimotodani et al, 2007b). Although MAFP also blocks fatty-acid amide hydrolase (FAAH), the enzyme that degrades another $\mathrm{eCB}$ anandamide, it is unlikely DSI is mediated by anandamide because the ability of MAFP to prolong DSI required DAGL activity. In addition, selective inhibitors of FAAH do not prolong DSI in other brain regions (Hashimotodani et al, 2007a).

\section{Stress-Induced Enhancement of DSI in the BLA}

Our biochemical data suggest that repeated restraint exposure results in adaptations in 2-AG metabolism that confers BLA neurons with an enhanced capacity to elevate 2-AG levels. We therefore tested the hypothesis that this increased capacity translates into an enhanced ability of BLA neurons to participate in eCB-mediated synaptic signaling. We examined DSI, an eCB-mediated form of short-term plasticity we showed to be mediated by $2-A G$, in control mice and those exposed to either 1 or 10 days of restraint stress. Our data indicate that acute $1 \mathrm{~h}$ restraint exposure does not effect DSI magnitude or duration compared with control mice, consistent with our biochemical data that acute restraint stress does not alter 2-AG or precursor DAG levels in the BLA. However, exposure to 10 days of restraint stress, which increased 2-AG and DAG levels, increased DSI duration, without effecting maximal DSI magnitude. Longer duration recordings at $0.33 \mathrm{~Hz}$ exhibited a run-down, thus we were not able to accurately determine the duration of DSI in stressed mice under these conditions. The duration of eIPSC depression in stressed mice remains an important open question. These findings support the hypothesis that the enhanced ability of BLA neurons to increase 2-AG levels after repeated stress exposure is associated with enhanced capacity for 2-AGmediated short-term synaptic suppression of GABAergic transmission in this region.

The increase in 2-AG that occurs in response to repeated restraint stress is transient, and subsides on termination of the stressor. Thus, it is unlikely that the enhanced DSI observed in stressed mice is directly related to stressinduced increases in 2-AG content because slice preparation and recording times were up to several hours after termination of the stressor; that is, it is unlikely that the 2-AG that was elevated during the restraint exposure was still present during recordings. Instead, we suggest that adaptations in one or more enzymatic components of 2-AG metabolism have occurred in response to repeated stress exposure conferring an enhanced capacity of BLA neurons from stressed mice to engage in 2-AG-mediated short-term synaptic plasticity in response to appropriate stimulation, in this case depolarization.

That repeated restraint stress and MAFP produce mutually occlusive prolongation of DSI suggests decreased 2-AG degradation may contribute to this effect. However, earlier studies showed no changes in MGL activity in response to 10 days of restraint stress (Rademacher et al, 2008), and we have not detected changes in MGL protein expression after 10 days of restraint stress (Patel and Winder, unpublished data). MGL contributes approximately $85 \%$ of the 2-AG hydrolytic activity in mouse brain, however, two other enzymes ABHD6 and ABHD12 contribute approximately 4 and 12\%, respectively (Blankman et al, 2007). It has been suggested that ABHD12 may preferentially be involved in the regulation of synaptically available 2-AG based on its extracellular hydrolytic activity (Blankman et al, 2007). The possibility that repeated restraint stress modulates the duration of 2-AG signaling through alterations $\mathrm{ABDH} 12$ rather than MGL remains to be tested.

Alternatively, it has been shown, in isolated rat BLA neurons, that late phase DSI is mediated by metabotropic glutamate receptor-5-driven eCB release, not calciummediated eCB release (Zhu and Lovinger, 2005). These data raise the possibility that repeated stress could be enhancing the receptor-driven late component of DSI, which is not present under control conditions. This could occur by an eCB 'priming' phenomenon which has recently been described by Alger and co-workers (Edwards et al, 2008). In hippocampal neurons, DSI can be enhanced by prior activation of group-1 metabotropic glutamate receptors. It is possible that repeated restraint stress progressively 'primes' neurons in vivo, and this priming is measurable ex vivo several hours later as enhanced late DSI. 
Finally, the enhanced DSI is not likely to be mediated through enhanced $C_{1}$ receptor sensitivity as our data indicate that $\mathrm{CB}_{1}$ receptor sensitivity to agonist is decreased, rather than increased by repeated restraint stress. These data are consistent with a recent report showing a decrease in $\mathrm{CB}_{1}$ receptor-mediated inhibition of GABA release in the striatum after 3 days of social defeat stress (Rossi et al, 2008). We have shown earlier that $\mathrm{CB}_{1}$ receptor number and affinity are unchanged in the amygdala after 10 days of restraint stress (Rademacher et al, 2008). These data seem discordant, however, earlier studies have shown that in vivo exposure to $\mathrm{CB}_{1}$ agonists can decrease $\mathrm{CB}_{1}$ receptor sensitivity without affecting receptor number or signaling efficacy (Mato et al, 2004). This form of 'functional tolerance' may be occurring in our model as a result of increased $C_{1}$ receptor activation by endogenous $2-A G$ in response to repeated restraint exposures. Further studies into the molecular determinants of stress-induced increases in 2-AG levels will be required to gain insight into the mechanisms responsible for enhanced $\mathrm{eCB}$ signaling observed in this study.

\section{Functional Implications}

BLA pyramidal neurons influence physiological, hormonal, and behavioral responses to stress in part through excitatory projections to the central extended amygdala, prefrontal cortex, and striatum (Sah et al, 2003). The spontaneous activity of these neurons is maintained at low levels by high local GABAergic inhibitory tone. Impairments in GABAergic transmission within the BLA have been suggested to contribute to anxiety and affective disorders (Quirk and Gehlert, 2003). $\mathrm{CB}_{1}$ receptors inhibit local GABAergic transmission within the BLA (Azad et al, 2003; Katona et al, 2001), an effect that likely contributes to the anxiogenic effects of high-dose exogenous cannabinoids (Patel et al, 2005a). Our data indicate that repeated stress enhances the ability of BLA neurons to engage in DSI, a form of short-term activity-dependent neuroplasticity that could enhance persistent firing of principle neurons (Carter and Wang, 2007). These data suggest that stress-induced enhancement in eCB signaling could result in increased activity of BLA pyramidal neurons, and contribute to the expression of physiological and behavioral responses to repeated stress. However, $\mathrm{CB}_{1}$ receptors also modulate excitatory transmission in the BLA (Azad et al, 2003), which could counteract the effects on GABAergic transmission. The balance between the effects of stress-induced increases in eCB signaling on GABAergic and glutamatergic transmission will determine the net influence of BLA neuron activity. Determining the effects of repeated stress on eCB regulation of excitatory transmission will be required to address this issue.

This study shows that repeated homotypic stress exerts metaplastic control of eCB-mediated short-term signaling in the BLA and provides further insight into the synaptic adaptations in eCB signaling that occur in response to repeated stress. Further behavioral and cellular studies into these adaptations may uncover novel approaches to the treatment of stress-related neuropsychiatric disorders, and help elucidate neural mechanisms subserving the interaction between stress and mental illness.

\section{ACKNOWLEDGEMENTS}

These studies were supported by the National Institutes of Health Grants DA19112, AA15635, DA11322, DA21696, and GM15431. SP was supported by the Vanderbilt University Department of Psychiatry, and a grant from the Vanderbilt Institute for Clinical and Translational Research.

\section{DISCLOSURE}

The authors declare no conflict of interest.

\section{REFERENCES}

Ahn K, McKinney MK, Cravatt BF (2008). Enzymatic pathways that regulate endocannabinoid signaling in the nervous system. Chem Rev 108: 1687-1707.

Azad SC, Eder M, Marsicano G, Lutz B, Zieglgansberger W, Rammes G (2003). Activation of the cannabinoid receptor type 1 decreases glutamatergic and GABAergic synaptic transmission in the lateral amygdala of the mouse. Learn Mem 10: 116-128.

Azad SC, Monory K, Marsicano G, Cravatt BF, Lutz B, Zieglgansberger W et al (2004). Circuitry for associative plasticity in the amygdala involves endocannabinoid signaling. J Neurosci 24: 9953-9961.

Blankman JL, Simon GM, Cravatt BF (2007). A comprehensive profile of brain enzymes that hydrolyze the endocannabinoid 2-arachidonoylglycerol. Chem Biol 14: 1347-1356.

Carter E, Wang XJ (2007). Cannabinoid-mediated disinhibition and working memory: dynamical interplay of multiple feedback mechanisms in a continuous attractor model of prefrontal cortex. Cereb Cortex 17(Suppl 1): i16-i26.

Caspi A, Sugden K, Moffitt TE, Taylor A, Craig IW, Harrington H et al (2003). Influence of life stress on depression: moderation by a polymorphism in the 5-HTT gene. Science 301: 386-389.

Edwards DA, Zhang L, Alger BE (2008). Metaplastic control of the endocannabinoid system at inhibitory synapses in hippocampus. Proc Natl Acad Sci USA 105: 8142-8147.

Hammen C (2005). Stress and depression. Annu Rev Clin Psychol 1: 293-319.

Hammen C, Brennan PA, Shih JH (2004). Family discord and stress predictors of depression and other disorders in adolescent children of depressed and nondepressed women. J Am Acad Child Adolesc Psychiatry 43: 994-1002.

Hashimotodani Y, Ohno-Shosaku T, Kano M (2007a). Ca(2+)assisted receptor-driven endocannabinoid release: mechanisms that associate presynaptic and postsynaptic activities. Curr Opin Neurobiol 17: 360-365.

Hashimotodani Y, Ohno-Shosaku T, Kano M (2007b). Presynaptic monoacylglycerol lipase activity determines basal endocannabinoid tone and terminates retrograde endocannabinoid signaling in the hippocampus. J Neurosci 27: 1211-1219.

Hill MN, Gorzalka BB (2005). Is there a role for the endocannabinoid system in the etiology and treatment of melancholic depression? Behav Pharmacol 16: 333-352.

Juhasz G, Chase D, Pegg E, Downey D, Toth ZG, Stones K et al (2009). CNR1 gene is associated with high neuroticism and low agreeableness and interacts with recent negative life events to predict current depressive symptoms. Neuropsychopharmacology 34: 2019-2027.

Jung KM, Astarita G, Zhu C, Wallace M, Mackie K, Piomelli D (2007). A key role for diacylglycerol lipase-alpha in metabotropic glutamate receptor-dependent endocannabinoid mobilization. Mol Pharmacol 72: 612-621.

Jung KM, Mangieri R, Stapleton C, Kim J, Fegley D, Wallace M et al (2005). Stimulation of endocannabinoid formation in brain slice 
cultures through activation of group I metabotropic glutamate receptors. Mol Pharmacol 68: 1196-1202.

Kamprath K, Marsicano G, Tang J, Monory K, Bisogno T, Di Marzo V et al (2006). Cannabinoid CB1 receptor mediates fear extinction via habituation-like processes. J Neurosci 26: 6677-6686.

Kano M, Ohno-Shosaku T, Hashimotodani Y, Uchigashima M, Watanabe M (2009). Endocannabinoid-mediated control of synaptic transmission. Physiol Rev 89: 309-380.

Katona I, Rancz EA, Acsady L, Ledent C, Mackie K, Hajos N et al (2001). Distribution of CB1 cannabinoid receptors in the amygdala and their role in the control of GABAergic transmission. J Neurosci 21: 9506-9518.

Katona I, Urban GM, Wallace M, Ledent C, Jung KM, Piomelli D et al (2006). Molecular composition of the endocannabinoid system at glutamatergic synapses. J Neurosci 26: 5628-5637.

Kreitzer AC, Regehr WG (2001). Cerebellar depolarization-induced suppression of inhibition is mediated by endogenous cannabinoids. J Neurosci 21: RC174.

Long JZ, Li W, Booker L, Burston JJ, Kinsey SG, Schlosburg JE et al (2009). Selective blockade of 2-arachidonoylglycerol hydrolysis produces cannabinoid behavioral effects. Nat Chem Biol 5: 37-44.

Marsicano G, Wotjak CT, Azad SC, Bisogno T, Rammes G, Cascio MG et al (2002). The endogenous cannabinoid system controls extinction of aversive memories. Nature 418: 530-534.

Mato S, Chevaleyre V, Robbe D, Pazos A, Castillo PE, Manzoni OJ (2004). A single in-vivo exposure to delta 9THC blocks endocannabinoid-mediated synaptic plasticity. Nat Neurosci 7: 585-586.

Melchior M, Caspi A, Milne BJ, Danese A, Poulton R, Moffitt TE (2007). Work stress precipitates depression and anxiety in young, working women and men. Psychol Med 37: 1119-1129.

Moreira FA, Lutz B (2008). The endocannabinoid system: emotion, learning and addiction. Addict Biol 13: 196-212.

Nomura DK, Hudak CS, Ward AM, Burston JJ, Issa RS, Fisher KJ et al (2008). Monoacylglycerol lipase regulates 2-arachidonoylglycerol action and arachidonic acid levels. Bioorg Med Chem Lett 18: 5875-5878.

Patel S, Cravatt BF, Hillard CJ (2005a). Synergistic interactions between cannabinoids and environmental stress in the activation of the central amygdala. Neuropsychopharmacology 30: 497-507.

Patel S, Hillard CJ (2003). Cannabinoid-induced Fos expression within A10 dopaminergic neurons. Brain Res 963: 15-25.
Patel S, Hillard CJ (2008). Adaptations in endocannabinoid signaling in response to repeated homotypic stress: a novel mechanism for stress habituation. Eur J Neurosci 27: 2821-2829.

Patel S, Roelke CT, Rademacher DJ, Hillard CJ (2005b). Inhibition of restraint stress-induced neural and behavioural activation by endogenous cannabinoid signalling. Eur J Neurosci 21: 1057-1069.

Piomelli D (2003). The molecular logic of endocannabinoid signalling. Nat Rev 4: 873-884.

Pittenger C, Duman RS (2008). Stress, depression, and neuroplasticity: a convergence of mechanisms. Neuropsychopharmaco$\log y$ 33: 88-109.

Quirk GJ, Gehlert DR (2003). Inhibition of the amygdala: key to pathological states? Ann N Y Acad Sci 985: 263-272.

Rademacher DJ, Meier SE, Shi L, Ho WS, Jarrahian A, Hillard CJ (2008). Effects of acute and repeated restraint stress on endocannabinoid content in the amygdala, ventral striatum, and medial prefrontal cortex in mice. Neuropharmacology 54: 108-116.

Rossi S, De Chiara V, Musella A, Kusayanagi H, Mataluni G, Bernardi $\mathrm{G}$ et al (2008). Chronic psychoemotional stress impairs cannabinoid-receptor-mediated control of GABA transmission in the striatum. J Neurosci 28: 7284-7292.

Sah P, Faber ES, Lopez De Armentia M, Power J (2003). The amygdaloid complex: anatomy and physiology. Physiol Rev 83: 803-834.

Uchigashima M, Narushima M, Fukaya M, Katona I, Kano M, Watanabe M (2007). Subcellular arrangement of molecules for 2-arachidonoyl-glycerol-mediated retrograde signaling and its physiological contribution to synaptic modulation in the striatum. J Neurosci 27: 3663-3676.

Viveros MP, Marco EM, File SE (2005). Endocannabinoid system and stress and anxiety responses. Pharmacol Biochem Behav 81: 331-342.

Wilson RI, Nicoll RA (2001). Endogenous cannabinoids mediate retrograde signalling at hippocampal synapses. Nature 410: 588-592.

Yamagata K, Andreasson KI, Kaufmann WE, Barnes CA, Worley PF (1993). Expression of a mitogen-inducible cyclooxygenase in brain neurons: regulation by synaptic activity and glucocorticoids. Neuron 11: 371-386.

Zhu PJ, Lovinger DM (2005). Retrograde endocannabinoid signaling in a postsynaptic neuron/synaptic bouton preparation from basolateral amygdala. J Neurosci 25: 6199-6207. 